\title{
Diversidad florística del bosque seco tropical en las subregiones bajo y medio Sinú, Córdoba, Colombia
}

\author{
Rosalba Ruiz V. ${ }^{*}$ \& Heidy Paola Saab R. ${ }^{1}$ \\ 1. Grupo de Investigación Botánica, Departamento de Biología, Universidad de Córdoba, Montería, Colombia; \\ rruizv@correo.unicordoba.edu.co, heidysaabr@correo.unicordoba.edu.co \\ * Correspondencia
}

Recibido 07-IX-2019. Corregido 19-XI-2019. Aceptado 21-I-2020.

\begin{abstract}
Floristic diversity of the tropical dry forest in the lower and middle Sinú subregion, Córdoba, Colombia. Introduction: The Tropical Dry Forest (TDF) is one of the most threatened ecosystems, in Colombia it has the greater extension in the Caribbean region, undergoing continuous transformation, causing the reduction of its coverage. Objective: To characterize the floristic diversity in terms of composition and structure in six TDF fragments in the lower and middle Sinú subregions of the Department of Córdoba. Methods: The methodology of Rapid Vegetation Sampling Plots - modified RAP was followed. Wealth and abundance were determined, The Importance Value Index by species and family, diametric and altimetric classes were constructed and the Fisher's $\alpha$ Diversity Index and Sørensen's Similarity Index were calculated. Results: 1336 individuals were registered, grouped into 68 families, 161 genera and 273 species in the middle Sinú and 2178 individuals (226 species / 151 genera / 59families) in the lower Sinú. The most important families by species richness were Fabaceae, Rubiaceae and Bignoniaceae, the highest abundance values were found in Fabaceae, Capparaceae and in the species Astronium graveolens Jacq. and Capparidastrum frondosum (Jacq.) Cornejo \& Iltis. The distribution by diametric and altimetric classes presented an inverted J behavior. The bushes represented the dominant growth habit. Attalea butyracea (Mutis ex L.f.) Wess.Boer, Cavanillesia platanifolia (Humb. \& Bonpl.) Kunth and Sterculia apetala (Jacq.) H.Karst. recorded the highest values of importance. Conclusions: The forests studied presented the floristic composition that characterizes the TDF in Colombia. The information obtained contributes to the integral management of the Tropical Dry Forest in Córdoba.
\end{abstract}

Key words: dry forest, Colombia, composition, Caribbean region, structure.

Ruiz V., R., \& Saab R., H.P. (2020). Diversidad florística del bosque seco tropical en las subregiones bajo y medio Sinú, Córdoba, Colombia. Revista de Biología Tropical, 68(1), $167-179$

El bosque seco tropical (Bs-T) representa cerca del $42 \%$ de los ecosistemas tropicales del mundo, se encuentra en tierras bajas de zonas tropicales y presenta una época de lluvias con varios meses de sequía, lo que confiere como característica principal el déficit de agua (Bullock, Mooney, \& Medina, 1995). En Colombia, de acuerdo a Etter, McAlpine y Possingham (2008), se registraba una extensión de 8882854 ha, entre mosaico de bosques y arbustales densos; actualmente se ha reducido a remanentes de bosques identificados en cuatro regiones biogeográficas, Caribe, Norandina, Valle del Cauca y Valle del Magdalena, con cerca de 720000 ha a escala 1:100000, de las cuales, 348416 (48.4\%) representan matrices de pastos, cultivos y espacios naturales y 332810 ha $(46.2 \%)$ corresponde a bosque seco natural, con la mayor extensión en la región Caribe, $28.1 \%$ (202423 ha), de las cuales, para 
el departamento de Córdoba se visualizan 1673 ha distribuidas hacia la parte norte (García, Corzo, Isaacs, \& Etter, 2014).

El bosque seco se ha clasificado en la categoría de estado crítico (Banda et al., 2016), debido a su larga historia de transformación, marcada por diferentes presiones como el cambio climático, la fragmentación y el fuego, la conversión agropecuaria por sus suelos fértiles y el establecimiento de asentamientos humanos, que a su vez demanda un crecimiento en la infraestructura vial, alterando la estructura y función del ecosistema (Miles et al., 2006).

La reducción de grandes masas de bosque seco en Colombia, se debe en gran parte a la falta de conocimientos sobre su dinámica e importancia en la generación de servicios ecosistémicos (Pizano \& García, 2014); en el departamento de Córdoba, la dinámica espacio temporal del bosque seco demuestra que las coberturas han disminuido significativamente (Ospino \& Ramos, 2014) y son pocos los esfuerzos fundamentados en conocer el estado actual y la distribución de la especies que caracterizan este ecosistema en la región, lo cual constituye la base en la gestión del conocimiento para garantizar un manejo integral del ecosistema.

Para Córdoba, la información asociada a la vegetación del bosque seco se remite a catálogos de flora realizados para la región Caribe (Rivera, 2010; Rivera \& Rangel, 2012; Rangel,
Cortes, \& Carvajal, 2012), proyectos que han centrado su objetivo en la recuperación de la vegetación adelantados por la Corporación Autónoma Regional de los Valles del Sinú y San Jorge (CVS) y comparación de aspectos florísticos del bosque seco relacionados a paisajes de ganadería extensiva y convencional (Ballesteros, Morelo, \& Pérez, 2019); por lo tanto, el objetivo del presente trabajo fue reconocer las especies presentes en el bosque seco tropical, determinando su composición y estructura en seis fragmentos distribuidos en las subregiones bajo y medio Sinú del departamento de Córdoba.

\section{MATERIALES Y MÉTODOS}

Área de estudio: El trabajo se desarrolló en el 2017 y 2018 en seis fragmentos de bosque seco tropical (Tabla 1) distribuidos en dos subregiones del departamento de Córdoba, ubicado en la región Caribe, hacia el extremo noroccidental del país, entre los $\left(09^{\circ} 26^{\prime} 16^{\prime \prime}\right.$ $07^{\circ} 22^{\prime} 0^{\prime \prime} \mathrm{N} \& 44^{\circ} 47^{\prime} 43^{\prime \prime}$ - 76 $\left.30^{\circ} 01^{\prime \prime} \mathrm{W}\right)$, con una superficie de $25020 \mathrm{~km}^{2}$, en las localidades de estudio predominan las actividades ganaderas y aplicación de cultivos transitorios, especialmente algodón, maíz y arroz, presenta un clima cálido tropical con temperatura promedio de $28{ }^{\circ} \mathrm{C}$ y precipitación promedio anual de 1200 - 1300 mm/año (Graciliano, Teobaldis, \& Combat, 2006).

TABLA 1

Ubicación geográfica de fragmentos de bosque seco en las subregiones bajo y medio Sinú en el Departamento de Córdoba, Colombia

TABLE 1

Geographic location of fragments of dry forest in the lower and middle Sinú subregions in the Department of Córdoba, Colombia

\begin{tabular}{|c|c|c|c|}
\hline Subregión & Localidad & Coordenadas Geográficas & Altitud (m) \\
\hline \multirow[t]{3}{*}{ Bajo Sinú } & Lorica-Los Gómez Finca Providencia & $09^{\circ} 03^{\prime} 54.4^{\prime \prime} \mathrm{N}-75^{\circ} 55^{\prime} 31.0^{\prime \prime} \mathrm{W}$ & 44 \\
\hline & Momil-Pueblecito, Finca La Gardenia & $09^{\circ} 16^{\prime} 44.1^{\prime \prime} \mathrm{N}-75^{\circ} 40^{\prime} 53.7^{\prime \prime} \mathrm{W}$ & 63 \\
\hline & Momil- Cerro El Mohán & $09^{\circ} 13^{\prime} 25.2^{\prime \prime} \mathrm{N}-75^{\circ} 39^{\prime} 53.4^{\prime \prime} \mathrm{W}$ & 76 \\
\hline \multirow[t]{3}{*}{ Medio Sinú } & Montería- Jaraquiel Finca Pensilvania & $08^{\circ} 40^{\prime} 05,6^{\prime \prime} \mathrm{N}-75^{\circ} 56^{\prime} 06,4^{\prime \prime} \mathrm{W}$ & 63 \\
\hline & Montería- Finca Santa Isabel & $08^{\circ} 34^{\prime} 24,5^{\prime \prime} \mathrm{N}-75^{\circ} 41^{\prime} 56,8^{\prime \prime} \mathrm{W}$ & 66 \\
\hline & Montería-Las Palomas Finca El Pino & $08^{\circ} 25^{\prime} 09^{\prime \prime} \mathrm{N}-76^{\circ} 03^{\prime} 24,8^{\prime \prime} \mathrm{W}$ & 65 \\
\hline
\end{tabular}


Fase de campo: Para el levantamiento de información vegetal en cada fragmento, se siguió la metodología de Parcelas de Muestreo Rápido de Vegetación - RAP modificada por ISA - JAUM (citado por Ariza, Toro, \& Medina, 2009), mediante el establecimiento de cinco parcelas rectangulares de 4 × $50 \mathrm{~m}$, abarcando un área de 0.1 ha. Las muestras se colectaron en el marco del permiso de recolección de especies silvestres de la diversidad biológica con fines de investigación científica no comercial - resolución 00914 de 2017 de la Agencia Nacional de Licencias Ambientales ANLA y se procesaron de acuerdo a técnicas convencionales de herborización. El material fue depositado y determinado en el Herbario de la Universidad de Córdoba - HUC, siguiendo el sistema de clasificación APG IV (2016).

Análisis de datos: Para estimar la diversidad se calculó la riqueza de especies y el Índice de Diversidad $\alpha$ de Fisher (Fisher, Corbet, \& Williams, 1943), además, se determinó la abundancia mediante el número de individuos por especie y por familia. Para comparar la composición florística o similitud entre los fragmentos, se calculó el Índice de Similaridad de Sørensen (Ss).

Para determinar la estructura florística se siguieron los tipos de hábitos de crecimiento descritos por Mendoza (1999) y se construyeron clases diamétricas y altimétricas. Para evaluar la importancia ecológica de las especies y familias de plantas, se analizaron los Índices de Valor de Importancia por especie (IVI) y por familia (VIF) (Mostacedo \& Fredericksen, 2000; Moreno, 2001).

\section{RESULTADOS}

Se presentan los datos de diversidad florística en términos de composición (Tabla 2) y estructura del bosque seco para cada una de las subregiones estudiadas.

En la subregión bajo Sinú, los fragmentos que presentaron la mayor diversidad en términos de riqueza específica fueron Los Gómez y Pueblecito. Las especies con mayor abundancia registradas fueron Astronium graveolens Jacq., seguida de Capparidastrum frondosum (Jacq.) Cornejo \& Iltis y Coursetia ferruginea (Kunth) Lavin. Los resultados del índice de diversidad $\alpha$ de Fisher para los fragmentos de la subregión bajo Sinú, confirman la mayor diversidad en los fragmentos de Pueblecito y Los Gómez, con poca diferencia (41.8 y 39.2 respectivamente), mientras el fragmento El Mohán presentó la diversidad más baja (18.6).

En la subregión medio Sinú, las especies con mayor abundancia registradas fueron Dolichandra sp., Hiraea reclinata Jacq. y Ruprechtia costata Meisn. El fragmento que presentó la mayor diversidad fue Santa Isabel, seguido de El Pino (Fisher's alpha: 63.25 y 38.9 respectivamente).

TABLA 2

Composición florística en las subregiones bajo y medio Sinú, Córdoba-Colombia

TABLE 2

Comparison of floristic richness in the lower and Middle Sinú subregions, Córdoba-Colombia

\begin{tabular}{|c|c|c|c|c|}
\hline Subregión / Localidad & $\mathrm{N}^{\circ}$ individuos & $\mathrm{N}^{\circ}$ familias & $\mathrm{N}^{\circ}$ Géneros & $\mathrm{N}^{\circ}$ especies \\
\hline Bajo Sinú & 2.178 & 59 & 151 & 226 \\
\hline Los Gómez & 776 & 38 & 86 & 119 \\
\hline Pueblecito & 661 & 44 & 79 & 118 \\
\hline El Mohán & 741 & 29 & 55 & 66 \\
\hline Medio Sinú & 1336 & 68 & 161 & 273 \\
\hline Santa Isabel & 571 & 50 & 100 & 147 \\
\hline El Pino & 353 & 45 & 80 & 108 \\
\hline Jaraquiel & 412 & 30 & 57 & 75 \\
\hline
\end{tabular}


TABLA 3

Comparación de la riqueza florística en las subregiones bajo y medio Sinú, Córdoba-Colombia

TABLE 3

Comparison of floristic richness in the lower and Middle Sinú subregions, Córdoba-Colombia

\begin{tabular}{cclcc}
\hline \multicolumn{2}{c}{ Número de especies } & \multicolumn{2}{c}{ Familias más diversas } & \multicolumn{2}{c}{ Número de individuos } \\
Bajo Sinú & Medio Sinú & \multicolumn{1}{c}{ Medio Sinú } \\
\hline 32 & 37 & Fabaceae & 339 & 138 \\
23 & 20 & Rubiaceae & 122 & 69 \\
12 & 6 & Sapindaceae & 99 & 16 \\
16 & 9 & Bignoniaceae & 128 & 21 \\
4 & 9 & Malvaceae & 23 & 27 \\
4 & 5 & Euphorbiaceae & 63 & 9 \\
5 & 6 & Lecythidaceae & 16 & 17 \\
2 & 5 & Myrtaceae & 72 & 55 \\
7 & 5 & Polygonaceae & 58 & 27 \\
7
\end{tabular}

En la Tabla 3 se relaciona la riqueza florística para cada una de las subregiones, indicando el número de especies y de individuos para las familias más diversas. Los géneros Machaerium, Trichilia, Randia y Casearía reúnen el mayor número de especies para el bajo Sinú, al igual que en el medio Sinú, excepto Randia, donde se registra también los géneros Paullinia y Serjania dentro de los más representativos.

La composición florística de los fragmentos en cada una de las subregiones muestra que en el bajo Sinú, Pueblecito y Los Gómez (29.5\% Índice de Sorensen), comparten el mayor número de especies, mientras que El Mohán y Pueblecito presentaron la más baja similitud (14.9\%); en el Sinú medio, Pensilvania y Santa Isabel (22.7 \%) comparten el mayor número de especies, mientras que El Pino y Santa Isabel presentaron la más baja similitud (15.3\%). Para toda la zona de estudio, sólo se comparte el $13 \%$ del total de las especies registradas; en relación a las especies exclusivas para el medio Sinú se presentaron 47 en el fragmento Jaraquiel, 106 en Santa Isabel y 68 en el Pino, y la subregión bajo Sinú presentó 42 especies exclusivas para El Mohán, 71 en Los Gómez y 73 en Pueblecito.

Estructura: En el medio y bajo Sinú, de acuerdo con el hábito de crecimiento, los arbustos presentaron la cobertura dominante. Los taxones con mayor altura fueron Attalea butyracea (Mutis ex L.f.) Wess.Boer (20 m) y Trichilia pleeana (A.Juss.) C.DC. (15 m) en el bajo Sinú y Cavanillesia platanifolia (Humb. \& Bonpl.) Kunth con $30 \mathrm{~m}$ en el medio Sinú.

Las distribuciones por clases diamétricas para las dos subregiones muestran que el número de individuos disminuye a medida que el DAP aumenta se presentaron en forma de $\mathrm{J}$ invertida (Fig. 1), este mismo comportamiento se observó en la distribución por clases altimétricas para los fragmentos del bajo Sinú, mientras que, en el medio Sinú, la mayoría de los individuos se ubican en rangos de 2 a $4 \mathrm{~m}$ de altura. (Fig. 2).

Índice de Valor de Importancia: Las especies con mayor importancia ecológica en la subregión bajo Sinú fueron: $A$. butyracea, $A$. graveolens, C. ferruginea, Bactris major Jacq., C. frondosum y Albizia niopoides (Benth.) Burkart (Tabla 4). Fabaceae y Arecaceae, presentaron los mayores valores de importancia (17.8 y $13.6 \%$ respectivamente), seguido de Rubiaceae (8.2\%), Anacardiaceae (7.7\%) y Capparaceae (6.2\%).

En el medio Sinú, se definió a Sterculia apetala (Jacq.) H.Karst. y C. platanifolia como las especies con mayor relevancia o 


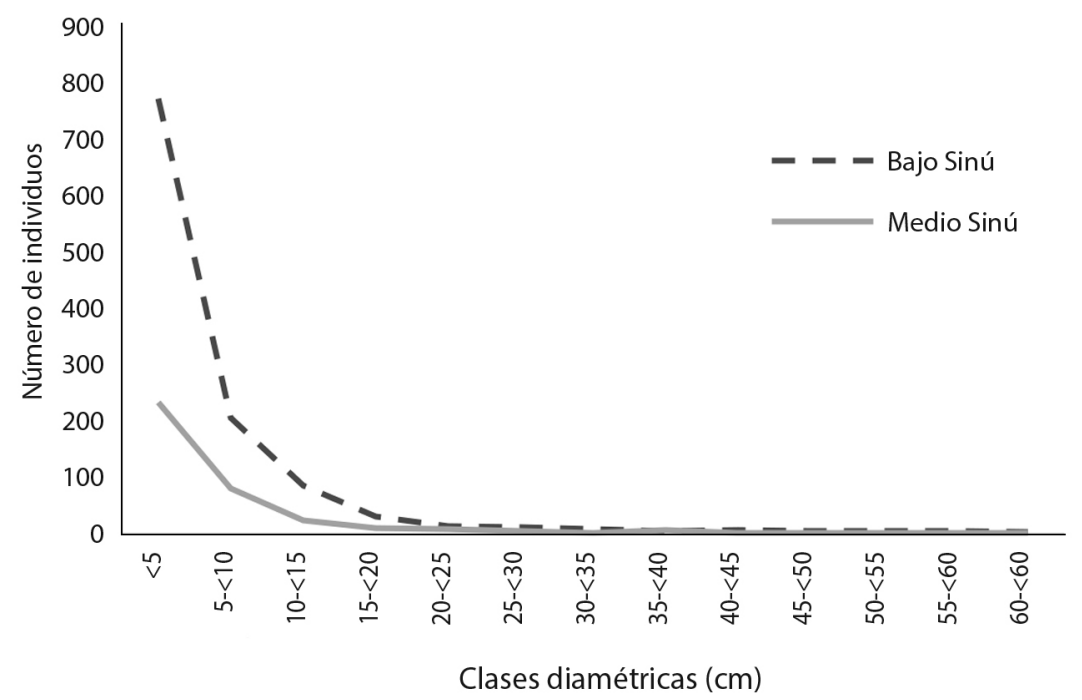

Fig. 1. Distribución diamétrica de individuos de bosque seco en las subregiones bajo y medio Sinú, Córdoba (Colombia). Fig. 1. Diametric distribution of dry forest individuals in the lower and middle Sinú subregions, Córdoba (Colombia).

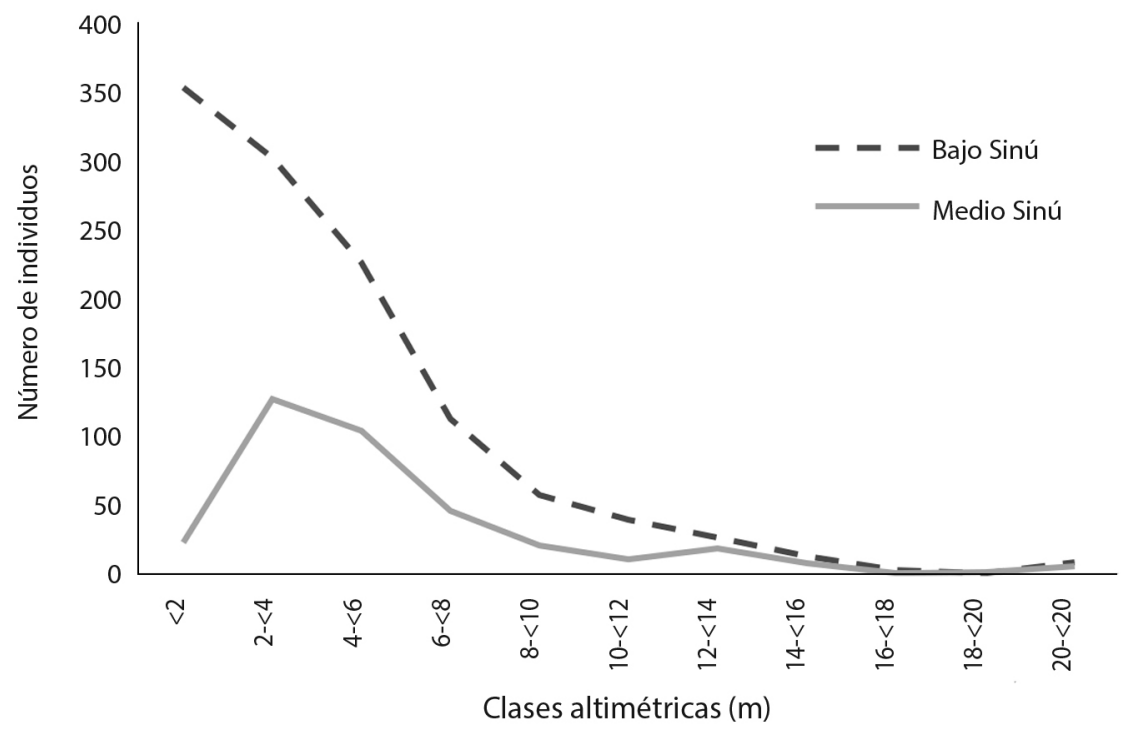

Fig. 2. Distribución altimétrica de individuos de bosque seco en las subregiones bajo y medio Sinú, Córdoba (Colombia). Fig. 2. Altimetric distribution of dry forest individuals in the lower and middle Sinú subregions, Córdoba (Colombia). 
TABLA 4

Especies con mayor Índice de Valor de Importancia-subregiones bajo y medio Sinú, Córdoba (Colombia)

TABLE 4

Species with the highest Value Index of Importance-lower and middle Sinú Subregion, Córdoba (Colombia)

\begin{tabular}{|c|c|c|c|c|c|c|c|}
\hline Especies & NI & DR & $\mathrm{AB}$ & DoR & FA & FR & IVI \\
\hline \multicolumn{8}{|l|}{ Bajo Sinú } \\
\hline Attalea butyracea & 14 & 1.23 & 2.51 & 29.06 & 2 & 1.11 & 10.47 \\
\hline Astronium graveolens & 118 & 10.40 & 0.88 & 10.27 & 2 & 1.11 & 7.26 \\
\hline Coursetia ferruginea & 59 & 5.20 & 0.49 & 5.76 & 1 & 0.55 & 3.84 \\
\hline Bactris major & 38 & 3.35 & 0.50 & 5.86 & 2 & 1.11 & 3.44 \\
\hline Capparidastrum frondosum & 72 & 6.34 & 0.04 & 0.51 & 2 & 1.117 & 2.65 \\
\hline Albizia niopoides & 25 & 2.20 & 0.310 & 3.58 & 2 & 1.117 & 2.303 \\
\hline Machaerium sp1. & 42 & 3.70 & 0.20 & 2.41 & 1 & 0.55 & 2.22 \\
\hline Eugenia sp1. & 57 & 5.02 & 0.04 & 0.48 & 1 & 0.55 & 2.02 \\
\hline Guazuma ulmifolia & 3 & 0.26 & 0.33 & 3.88 & 2 & 1.11 & 1.75 \\
\hline Trichilia pleeana & 32 & 2.82 & 0.10 & 1.20 & 2 & 1.11 & 1.71 \\
\hline Sub Total & 460 & 40.6 & 5.4 & 63.1 & 17 & 9.5 & 37.7 \\
\hline Especies restantes (136) & 674 & 59.4 & 3.2 & 36.9 & 162 & 90.5 & 62.3 \\
\hline Total & 1134 & 100 & 8.63 & 100 & 179 & 100 & 100 \\
\hline \multicolumn{8}{|l|}{ Medio Sinú } \\
\hline Sterculia apetala & 2 & 0.56 & 2.29 & 40.50 & 1 & 0.57 & 13.88 \\
\hline Cavanillesia platanifolia & 4 & 1.12 & 1.18 & 20.81 & 2 & 1.15 & 7.69 \\
\hline Ruprechtia costata & 33 & 9.24 & 0.03 & 0.55 & 1 & 0.57 & 3.46 \\
\hline Vitex cymosa & 1 & 0.28 & 0.40 & 7.10 & 1 & 0.57 & 2.65 \\
\hline Alchornea sp. & 1 & 0.28 & 0.29 & 5.12 & 1 & 0.57 & 1.99 \\
\hline Trichilia acuminata & 14 & 3.92 & 0.05 & 1.01 & 1 & 0.57 & 1.83 \\
\hline Faramea occidentalis & 15 & 4.20 & 0.01 & 0.13 & 2 & 1.15 & 1.83 \\
\hline Guazuma ulmifolia & 10 & 2.80 & 0.03 & 0.65 & 2 & 1.15 & 1.53 \\
\hline Pogonopus speciosus & 11 & 3.08 & 0.01 & 0.32 & 2 & 1.15 & 1.52 \\
\hline Casearia sylvestris & 7 & 1.96 & 0.11 & 1.95 & 1 & 0.57 & 1.49 \\
\hline Sub Total & 98 & 27.4 & 4.43 & 78.18 & 14 & 8.09 & 37.90 \\
\hline Especies Restantes & 259 & 72.5 & 1.23 & 21.81 & 159 & 91.90 & 62.09 \\
\hline Total & 357 & 100 & 5.670 & 100 & 173 & 100 & 100 \\
\hline
\end{tabular}

NI: Número de individuos; DR: Densidad relativa (\%); FA: Frecuencia absoluta; FR: Frecuencia relativa (\%); AB: Área basal; DoR: Dominancia relativa (\%); IVI: Índice de Valor de Importancia.

peso ecológico, seguidas de Ruprechtia costata Meisn., Vitex cymosa Bertero ex Spreng. y Alchornea sp. (Tabla 4) y Malvaceae, Rubiaceae, Fabaceae y Polygonaceae como las familias de mayor importancia ecológica concentrando el $48.8 \%$ del IVIF total.

\section{DISCUSIÓN}

En los bosques secos del departamento de Córdoba se encontró una alta diversidad vegetal a pesar de su estado crítico de fragmentación, la alta fertilidad de los suelos reportada para la región Caribe y el efecto del recambio de especies que se presenta dentro y entre regiones del bosque seco de Colombia (González et al., 2018), contribuyen a soportar la riqueza de estos bosques neotropicales; se comparten especies reconocidas como ecológicamente versátiles y generalistas de acuerdo a los 23 núcleos florísticos caracterizados por LinaresPalomino, Oliveira-Filho y Pennington (2011) 
para el bosque seco del Neotrópico, como Randia armata (Sw.) DC., Spondias mombin L., Maclura tinctoria (L.) D.Don ex Steud., Sapindus saponaria L., Sapium glandulosum (L.) Morong, Handroanthus ochraceus (Cham.) Mattos, Trema micrantha (L.) Blume, Trichilia pallida (Humb. \& Bonpl. ex Schult.) C.DC., Hymenaea courbaril L., Genipa americana L., Cochlospermum vitifolium (Willd.) Spreng., Casearia sylvestris Sw., Trichilia hirta L., Guazuma ulmifolia Lam., Cynophalla flexuosa (L.) J.Presl y Apeiba tibourbou Aubl.

La familia de mayor representación en términos de composición florística, fue Fabaceae, la cual predomina en los bosques secos de Colombia y reconocida como el grupo taxonómico más representativo, con amplia distribución y diversidad en los trópicos (Gentry, 1995; Instituto Alexander von Humboldt - IavH, 1998; Mendoza, 1999). En la zona de estudio dominaron los arbustos y los árboles, entre las lianas se destacó Bauhinia hymenaeifolia Hemsl., B. glabra y Phaseolus lunatus L., mientras que en las hierbas sólo se registró Dioclea $\mathrm{sp}$. La funcionalidad de esta familia es clave en procesos de restauración del bosque seco tropical, debido a su capacidad de colonización, fácil propagación, altas tasas de crecimiento y alta capacidad de fijar nitrógeno (Vargas \& Ramírez, 2014); Albizia guachapele (Kunth) Dugand, A. niopoides, G. sepium, Machaerium biovulatum Micheli, Myrospermum frutescens Jacq., Inga edulis Mart., Platymiscium pinnatum (Jacq.) Dugand y Pithecellobium lanceolatum (Willd.) Benth., corresponden a especies arbóreas claves en la recuperación de suelos y áreas degradadas por las actividades agropecuarias, que por su resistencia a épocas de sequía, se observan comúnmente en la zona de estudio como cercas vivas, delimitación de linderos y árboles dispersos en potreros para sombra y refugio del ganado; el establecimiento de estas especies se ve favorecido principalmente en la subregión del bajo Sinú, por la textura franco arcillosa que caracteriza a sus suelos, con variaciones del $\mathrm{pH}$ desde 6.3 para el fragmento de bosque en Pueblecito y 7.8 para el fragmento del Cerro
El Mohán, valores que se ajustan a lo reportado por González et al. (2018) para el Caribe, indicando además, que los altos valores de $\mathrm{pH}$ del suelo, caracterizado por una alta fertilidad $(\mathrm{pH}>6.3)$, factor asociado probablemente a la composición florística de los bosques secos de esta región.

Las subregiones medio y bajo Sinú del departamento de Córdoba, comparten el $45 \%$ de las especies encontradas por Mendoza (1999) para otras regiones del Caribe y el Valle del rio Magdalena, en muestreos de 0.1 ha y se mantiene el registro de las especies $B$. simaruba, C. peltata, G. sepium, Melicoccus bijugatus Jacq., P. lanceolatum, S. mombin, Chomelia spinosa Jacq., Cissus verticillata (L.) Nicolson \& C.E.Jarvis, Randia armata (Sw.) DC., Senna obtusifolia (L.) H.S. Irwin \& Barneby, Commelina erecta L., Petiveria alliacea L., Rivina humilis L. y Sida acuta Burm.f., reconocidas por Pizano et al. (2014) como las más frecuentes para la región Caribe.

Los valores de riqueza encontrados (403 spp.) son similares a lo reportado por Interconexión Eléctrica S. A.- ISA (2002) para seis localidades del departamento del Atlántico, en el norte de Colombia, con 428 especies y escasamente difiere de la riqueza encontrada por Herazo, Mercado y Mendoza (2017) en siete localidades de los Montes de María, en el departamento de Sucre, también en el norte de Colombia (363 spp.), con el cual, comparte mayor proporción en cuanto a su composición y estructura florística.

La familia Rubiaceae ocupa el segundo lugar en términos de riqueza, manteniendo los patrones de composición florística propuestos por Gentry (1995) para los bosques secos neotropicales, con distribución en diferentes ecosistemas (Villareal et al., 2004), y mejor representación en la región Caribe colombiana (Mendoza, 1999), en este trabajo se registró la más alta representación de Rubiaceae de bosques secos para el departamento de Córdoba, principalmente en los fragmento de Pueblecito y Santa Isabel, en comparación con lo descrito por Ballesteros, Morelo y Pérez (2019) para localidades de bosque seco en 
paisajes de ganadería extensiva bajo manejo silvopastoril y convencional en Córdoba, donde reportan 19 especies.

Los géneros encontrados con el mayor número de especies, coinciden generalmente a lo reportado para los bosques secos de la región Caribe y el valle del rio Magdalena (Marulanda et al., 2003; Mendoza, 1999), donde se reconocen a Capparis, Trichilia, Machaerium, Casearia, Bauhinia, Coccoloba, Aspidosperma, Eugenia, Randia, Paullinia y Tabebuia como los géneros más diversos.

Las especies del género Machaerium, logran desarrollarse en ecosistemas intervenidos y presentan mecanismo de dispersión de semillas por anemocoria, relacionada con la morfología de sus frutos tipo legumbre samaroide (Meléndez, 2009), favoreciendo su propagación en ambientes secos característicos de la zona de estudio, donde se utilizan en la recuperación y enriquecimiento de suelos por ser especies fijadoras de nitrógeno. Las siete especies encontradas para este género coincide con lo reportado por Mendoza (1999) para el bosque seco tropical del Valle del rio Magdalena y algunas localidades del Caribe; Herazo et al. (2017) mencionan a Machaerium como el género de mayor riqueza para los Montes de María en Sucre, así como lo reportan Carrillo, Rivera y Sánchez (2007) para el Cerro Tasajero en el municipio de Cúcuta (noreste de Colombia) donde se comparte M. arboreum (Jacq.) Vogel, M. microphyllum (E.Mey.) Standl. y M. biovulatum; Sanmartín, Angarita y Mercado (2016) reportan igualmente este género como el más rico para la Reserva Natural Sanguaré en Sucre, de las cuales se comparte también $M$. arboreum y M. microphyllum.

El género Serjania en la subregión del medio Sinú, presentó un mayor número de especies en comparación con otros bosques de la región Caribe, donde generalmente no se relaciona entre los taxones más diversos de este ecosistema, sin embargo, García y Rivera (2009) reportan a Serjania entre los géneros mejor representados con tres especies para un bosque seco en Aguachica, Cesar, al noreste de Colombia y se caracteriza por su resistencia a las quemas y condiciones de sequía (Gillespie, Grijalva, \& Farris, 2000; Vásquez, Pezo, Mora, \& Skarpe, 2012), como Serjania atrolineata Radlk., con distribución desde México a Venezuela, Bolivia y en la región Caribe colombiana.

El bosque seco de Córdoba también presenta afinidades florísticas con Mesoamérica especialmente con Costa Rica, compartiendo el $70 \%$ de géneros y $62 \%$ de las especies de plantas leñosas reportadas por Kalacska et al. (2004), al igual que las especies más comunes registradas por Gillespie et al. (2000) para Costa Rica y Nicaragua como Acacia collinsii Saff., Adenocalymma inundatum Mart. ex DC., Bauhinia glabra Jacq., Bombacopsis quinata (Jacq.) Dugand, Bursera simaruba (L.) Sarg., Handroanthus ochraceus (Cham.) Mattos, Calycophyllum candidissimum (Vahl) DC., Casearia corymbosa Kunth, Cecropia peltata L., C. vitifolium, Cordia alliodora (Ruiz \& Pav.) Oken, Bignonia diversifolia Kunth, Gliricidia sepium (Jacq.) Walp., Guazuma ulmifolia, Gyrocarpus americanus Jacq. y $A$. graveolens, esta última corresponde igualmente a la especie de mayor abundancia en la zona de estudio, lo cual se ha reportado para fragmentos de bosque seco en la región Caribe, en Toluviejo (Departamento de Sucre) (Olascuaga, Mercado, \& Sánchez, 2015), Tierra Bomba y Forestal Monterrey (Departamento de Bolívar) (Mendoza, 1999).

La mayor abundancia de $A$. graveolens se encontró en El Mohán (bajo Sinú), localidad que presenta las condiciones ambientales más favorables para el crecimiento de este elemento, son sitios no inundables, con suelos bien drenados y porcentajes de arcilla del $35 \%$, estos árboles pueden tolerar hasta seis meses de sequía al año (Unión Internacional para la Conservación de la Naturaleza-UICN, 2015), son resistentes al déficit de agua típico del bosque seco, además, presentan un crecimiento rápido, siendo considerada como especie pionera (Vargas, 2015), utilizada en procesos de restauración, cercas vivas y sombra para cultivos, actividades que favorecen su propagación por las comunidades aledañas dedicadas a actividades agropecuarias. 
Capparidastrum frondossum (Capparidaceae) se ubica como la segunda especie más abundante, con distribución en las dos subregiones, son arbustos que crecen en bosques intervenidos (Rodríguez, Banda, Reyes, \& Estupiñan, 2012). La mayoría de las especies de esta familia son perennifolias y llegan a ser dominantes en el bosque seco, por lo que representan también uno de los principales elementos en la composición florística de este ecosistema en el neotrópico (Aguirre \& Ruiz, 2017), además pueden ser potencialmente útiles en procesos de reforestación de zonas secas (Cornejo \& Iltis, 2012).

Los mayores valores de riqueza encontrados en la subregión del Sinú medio, se puede explicar probablemente por las condiciones ambientales de los fragmentos, especialmente su ubicación en proximidades del Rio Sinú con influencia de pequeños cuerpos de agua, favoreciendo el desarrollo de las especies (Herazo et al., 2017). La familia Bignoniaceae tuvo mayor representación en esta zona, con altos valores de abundancia para Dolichandra sp., reconocido como un grupo de lianas neotropicales que se distribuye ampliamente en diferentes hábitats y es un componente esencial de los bosques estacionalmente secos, con diversos sistemas de polinización y continuos cambios morfológicos florales asociados a eventos de polinización y dispersión como factor principal que moldeo la distribución geográfica del género (Fonseca \& Lohmann, 2015).

Con relación a la riqueza por localidades, el fragmento que presentó la mayor diversidad fue Santa Isabel, el cual, en términos de área no corresponde al de mayor tamaño, sin embargo, es el fragmento que se encuentra más aislado y con menor influencia de actividades agropecuarias. La diversidad registrada en Santa Isabel, puede estar asociada a la participación eventual de la corporación Autónoma Regional de los Valles del Sinú y San Jorge - CVS, en los procesos de recuperación del bosque realizados en esta zona con actividades de reforestación y liberación de especies de fauna silvestre, en el marco de la resolución 026 de julio 2012 (http://runap.parquesnacionales.gov. co/area-protegida/691), por medio de la cual se declaró el bosque de Santa Isabel como Reserva Natural de la Sociedad Civil, bajo la autoridad ambiental de Parques Nacionales Naturales de Colombia. La menor diversidad del fragmento el Mohán se debe posiblemente a la abundancia de especies como A. graveolens en esta localidad.

La distribución por clases diamétricas en forma de $\mathrm{J}$ invertida que se presentó en los fragmentos, indican que son bosques en recuperación (Uslar, Mostacedo, \& Saldias, 2004), como resultado del sometimiento a los efectos de la agricultura y la ganadería que predominan en el área; con relación a la distribución altimétrica, la mayoría de individuos se agrupó en las primeras clases de altura, entre los 2-4 m, como consecuencia de las perturbaciones por tala selectiva y entresaca de especies leñosas para uso maderable, combustible y de construcción, utilizando árboles como maderas para establos o estacones para delimitar potreros y cultivos en la zona, el hábito de crecimiento arbustivo fue el más frecuente, igual a lo reportado por Carrillo et al. (2007) y Herazo et al. (2017) para fragmentos de bosque seco tropical en el Cerro tasajero - Cúcuta y los Montes de María - Sucre respectivamente.

El mayor valor de importancia para la familia Malvaceae y consecuentemente para Sterculia apetala y Cavanillesia platanifolia en la subregión medio Sinú, se refleja por los altos valores de área basal, ya que se registraron muy pocos individuos que presentaron los valores más altos de DAP y las mayores alturas, son especies del dosel o árboles emergentes con amplia distribución para los bosques secos tropicales. C. platanifolia se considera una especie de etapas secundarias tempranas (Vargas, González, Barona, \& Bolívar, 2016), así como $S$. apetala, que también es fundamental en la restauración del ecosistema debido a su establecimiento en sucesiones secundarias de áreas en regeneración o en los claros del bosque, las plántulas se desarrollan tanto en ambientes conservados como alterados; sin embargo, es extraída del bosque por la calidad 
de su madera para construcción, ebanistería y leña (Fajardo, 2013).

En la subregión bajo Sinú, se mantiene la tendencia de dominar la familia Fabaceae con el mayor peso ecológico, seguida de Arecaceae, pero se destaca la mayor importancia ecológica registrada para la especie Attalea butyracea, esta palma presenta amplia distribución y es frecuente en todas las zonas secas del Caribe colombiano, distintiva de áreas perturbadas y en potreros, con frutos dispersados por el ganado vacuno, siendo la disponibilidad de luz fundamental para su crecimiento (Uribe, Velásquez, \& Montoya, 2001; Galeano \& Olivares, 2013; Olivares \& Galeano, 2013). La localidad con el mayor número de individuos de esta especie fue el bosque de Pueblecito en el municipio de Momil, donde crece en sitios abiertos con alta intensidad lumínica y se encuentra sometida a quemas para establecimiento de pastos, es utilizada por las poblaciones aledañas como ornamental, comestible, en actividades culturales y de construcción. Para bosques secos en el departamento de Sucre se reporta también una alta representación de palmas, siendo $A$. butyracea la especie más importante y Arecaceae la tercera familia con mayor valor de índice de importancia (Sanmartín et al., 2016).

La composición de especies encontrada en los bosques secos de Córdoba, indican que los fragmentos tienen poca similitud, comparten un porcentaje bajo de especies, en los fragmentos de la subregión medio Sinú sólo se registraron cinco especies comunes ( $A$. graveolens, M. microphyllum, Piper aduncum L., P. marginatum Jacq. y Solanum hirtum Vahl) y cuatro en el bajo Sinú (Casearia aculeata Jacq., Dolichandra uncata (Andrews) L.G.Lohmann, Pithecellobium hymenaeafolium (Willd.) Benth. y $R$. armata); lo anterior permite inferir que el aislamiento geográfico de los sitios estudiados en cada subregión y la falta de corredores biológicos que han limitado la conectividad entre los fragmentos, así como las condiciones microclimáticas debido a la topografía de los sitios y la intervención humana, son factores determinantes en el paisaje y estado actual de la vegetación.
Declaración de ética: los autores declaran que todos están de acuerdo con esta publicación y que han hecho aportes que justifican su autoría; que no hay conflicto de interés de ningún tipo; y que han cumplido con todos los requisitos y procedimientos éticos y legales pertinentes. Todas las fuentes de financiamiento se detallan plena y claramente en la sección de agradecimientos. El respectivo documento legal firmado se encuentra en los archivos de la revista.

\section{AGRADECIMIENTOS}

A la Universidad de Córdoba - Vicerrectoría de Investigación y Extensión por el financiamiento del proyecto "Estado de la diversidad florística y fragmentación del bosque seco tropical (Bs-T) en el bajo y medio Sinú, Departamento de Córdoba-Colombia, Al herbario de la Universidad de Córdoba - HUC por el apoyo logístico, a Álvaro Cogollo por su contribución en la determinación de material vegetal, a William, Pablo y Santiago por su acompañamiento en campo, a Danilo Nuñez, Jean Varilla, Aldair Arizal, Maria J. Atehortúa, Julieth Mestra, Fray Galarcio, Juan P. Chica, Jhon Reinel, Hector Furnieles y Sania Padilla por su apoyo en el campo.

\section{RESUMEN}

Introducción: El bosque seco tropical (Bs-T) es uno de los ecosistemas más amenazados, en Colombia, presenta la mayor extensión en la región Caribe, está sometido a continua transformación, que ha ocasionado la disminución de su cobertura. Objetivo: Caracterizar la diversidad florística en términos de composición y estructura en seis fragmentos de Bs-T en las subregiones bajo y medio Sinú del Departamento de Córdoba. Métodología: Se siguió la metodología de Parcelas de Muestreo Rápido de Vegetación - RAP modificada. Se determinó la riqueza y abundancia, el Índice de valor de Importancia por especie y por familia, se construyeron clases diamétricas y altimétricas y se calculó el Índice de Diversidad $\alpha$ de Fisher e Índice de Similaridad de Sørensen. Resultados: Se registraron 1336 individuos, agrupados en 68 familias, 161 géneros y 273 especies en el medio Sinú y 2178 individuos (226 especies/151 géneros/59 familias) en el bajo Sinú. Las familias más importantes por riqueza de especies fueron: Fabaceae, Rubiaceae y Bignoniaceae, los 
mayores valores de abundancia se presentaron en Fabaceae, Capparaceae y en las especies: Astronium graveolens Jacq. y Capparidastrum frondosum (Jacq.) Cornejo \& Iltis. La distribución por clases diamétricas y altimétricas presentó un comportamiento de $\mathrm{J}$ invertida. Los arbustos representaron el hábito de crecimiento dominante. Attalea butyracea (Mutis ex L.f.) Wess.Boer, Cavanillesia platanifolia (Humb. \& Bonpl.) y Sterculia apetala Sterculia apetala (Jacq.) H.Karst. registraron los mayores valores de importancia. Conclusiones: Los bosques estudiados presentaron la composición florística que caracteriza el Bs-T en Colombia. La información obtenida contribuye a la gestión integral del bosque seco en Córdoba.

Palabras clave: bosque seco, Colombia, composición, región Caribe, estructura.

\section{REFERENCIAS}

Aguirre, A., \& Ruiz, T. (2017). Capparaceae Juss. del departamento del Atlántico, Colombia. Ciencia en Desarrollo, 8(1), 51-69.

Angiosperm Phylogeny Group - APG. (2016). An update of the Angiosperm Phylogeny Group classification for the orders and families of flowering plants: APG IV. Botanical Journal of the Linnean Society, 181, 1-20.

Ariza, W., Toro, J., \& Medina, A. (2009). Análisis florístico y estructural de los bosques premontanos en el municipio de Amalfi (Antioquia, Colombia). Colombia Forestal, 12, 81-102.

Ballesteros, J., Morelo, L., \& Pérez, J. (2019). Composición y estructura vegetal de fragmentos de bosque seco tropical en paisajes de ganadería extensiva bajo manejo silvopastoril y convencional en Córdoba, Colombia. Caldasia, 41(1), 224-234.

Banda, K., Delgado, A., Dexter, K., Linares, R., Oliveira, A., Prado, D., ... Pennington, R. (2016). Plant diversity patterns in neotropical dry forests and their conservation implications. Science, 353(6306), $1383-1387$

Bullock, S., Mooney, H., \& Medina, E. (Eds.). (1995). Seasonally Dry Tropical Forests. Cambridge, Inglaterra: Cambridge University Press.

Carrillo, M., Rivera, O., \& Sánchez, R. (2007). Caracterización florística y estructural del Bosque seco Tropical del Cerro Tasajero, San José de Cúcuta (Norte de Santander), Colombia. Actualidades Biológicas, 29(86), 55-73.

Cornejo, X., \& Iltis, H. H. (2012). Flora de Jalisco y áreas colindantes: Capparaceae. Guadalajara, México: Orgánica Editores.

Etter, A., McAlpine, C., \& Possingham, H. (2008). Historical Patterns and Drivers of Landscape change in
Colombia since 1500: A regionalized spatial approach. Annals of the Association of American Geographers, 98(1), 2-23.

Fajarado, F. (2013). Interacción entre las semillas de Sterculia apetala (Jacq.) H. Karst. y hemípteros del género Dysdercus en el Jardín Botánico Guillermo Piñeres de Cartagena (Tesis de Maestría). Universidad Nacional de Colombia, Bogotá.

Fisher, R., Corbet, S., \& Williams, C. (1943). The relation between the number of species and the number of individuals in a random sample of an animal population. Journal Animal Ecology, 12(1), 42-58.

Fonseca, L. H. M., \& Lohmann, L. G. (2015). Biogeography and evolution of Dolichandra (Bignonieae, Bignoniaceae). Botanical Journal of the Linnean Society, 179(3), 403-420.

Galeano, G., \& Olivares, I. (2013). Palma de vino (Attalea butyracea). En R. Bernal \& G. Galeano (Eds.), Cosechar sin destruir - Aprovechamiento sostenible de palmas colombianas (pp. 166-174). Bogotá, Colombia: Panamericana Formas e Impresos S.A.

García, H., Corzo, G., Isaacs, P., \& Etter, A. (2014). Distribución y Estado Actual de los Remanentes del Bioma de Bosque Seco Tropical en Colombia: Insumos para su gestión. En C. Pizano \& H. García (Eds.), El Bosque Seco Tropical en Colombia (pp. 229-251). Bogotá, Colombia: Ediprint Ltda.

García, J., \& Rivera, O. (2009). Composición florística del bosque El Agüil (Aguachica, Cesar) con anotaciones sobre su estructura. En J. O. Rangel (Ed.), Colombia Diversidad Biótica VIII: Media y baja montaña de la serranía de Perijá (pp. 575-601). Bogotá, Colombia: Universidad Nacional de Colombia.

Gentry, A. (1995). Diversity and floristic composition of neotropical dry forests. In S. Bullock, H. Mooney, \& E. Medina (Eds.), Seasonally Dry Tropical Forests (pp. 146-194). Cambridge, Inglaterra: Cambridge University Press.

Gillespie, T., Grijalva, A., \& Farris, C. (2000). Diversity, composition, and structure of tropical dry forests in Central America. Plant Ecology, 147, 37-47.

González-M. R., García, H., Isaacs, P., Tablas, H., López, R., Rodríguez, N., ... Pizano, C. (2018). Disentangling the environmental heterogeneity, floristic distinctiveness and current threats of tropical dry forests in Colombia. Environmental Research Letters, $13,1-12$.

Graciliano, P., Teobaldis, M., \& Combat, E. (2006). Estudio Agroclimático del Departamento de Córdoba. Montería, Colombia: Fondo Editorial Universidad de Córdoba.

Herazo, F., Mercado, J., \& Mendoza, H. (2017). Estructura y Composición Florística del Bosque Seco Tropical 
en los Montes de María (Sucre - Colombia). Ciencia en Desarrollo, 8(1), 71-82.

Instituto de Investigación Alexander von Humboldt - IAvH. (1998). El bosque seco tropical en Colombia. Recuperado de http://media.utp.edu.co/ciebreg/archivos/ bosque-seco-tropical/el-bosque-seco-tropical-encolombia.pdf

Interconexión Eléctrica S. A.-ISA. (2002). Inventario de flora, fauna y establecimiento del programa de monitoreo en áreas de jurisdicción de Cardique y Cra. Informe Técnico. Cartagena, Colombia: ISA, Fundación Jardín Botánico Joaquín Antonio Uribe de Medellín.

Kalacskaa, M., Sanchez-Azofeifaa, G. A., Calvo-Alvaradob, J. C., Quesadac, M., Rivarda, B., \& Jansen, D. H. (2004). Species composition, similarity and diversity in three successional stages of a seasonally dry tropical forest. Forest Ecology and Management, 200, 227-247.

Linares-Palomino, R., Oliveira-Filho, A., \& Pennington, R. T. (2011). Neotropical seasonally dry forests: Diversity, endemism, and biogeography of woody plants. In R. Dirzo, H. S. Young, H. A. Mooney, \& G. Ceballos (Eds.), Seasonally Dry Tropical Forests: Ecology and Conservation (pp. 3-21). Washington, Estados Unidos: Island Press.

Marulanda, A., Uribe, P., Velásquez, M., Montoya, A., Idárraga, A., López, M. C., \& López, L. (2003). Estructura y composición de la vegetación de un fragmento de bosque seco en San Sabastián, Magdalena (Colombia). I. Composición de plantas vasculares. Actualidades Biológicas, 25(78), 17-30.

Meléndez-G. P. (2009). Synopsis of the genus Machaerium Pers. (LeguminosaePapilionoideae-Dalbergieae) in Venezuela. Acta Botánica Venezuelica, 32(2), 363-416.

Mendoza, H. (1999). Estructura y riqueza florística del bosque seco tropical en la región Caribe y el valle del río Magdalena, Colombia. Caldasia, 21(1), 70-94.

Miles, L., Newton, A. C., DeFries, R. S., Ravilious, C., May, I., Blyth, S., Kapos, V., \& Gordon, J. E. (2006). A global overview of the conservation status of tropical dry forests. Journal of Biogeography, 33(3), 491-505.

Moreno, C. (2001). Métodos para medir la biodiversidad (M \& T-Manuales y Tesis SEA, vol. I). Zaragosa, España: Gorfi, S.A.

Mostacedo, B., \& Fredericksen, T. (2000). Manual de Métodos Básicos de Muestreo y Análisis en Ecología Vegetal. Proyecto de Manejo Forestal Sostenible (BOLFOR). Santa Cruz de la Sierra, Bolivia: Editora El País.
Olascuaga, D., Mercado, J., \& Sánchez, L. (2015). Análisis de la vegetación sucesional en un fragmento de bosque seco tropical en Toluviejo-Sucre (Colombia). Colombia Forestal, 19(1), 23-40.

Olivares, I., \& Galeano, G. (2013). Leaf and inflorescence production of the wine palm (Attalea butyracea) in the dry Magdalena river valley, Colombia. Caldasia, $35(1), 37-48$

Ospino, J. C., \& Ramos, A. (2014). Análisis geográfico de la pérdida y/o fragmentación del bosque seco tropical en Córdoba mediante imágenes landsat para el periodo 1985-2013 (Tesis de pregrado). Universidad de Córdoba, Montería, Colombia.

Pizano, C., \& García, H. (Eds.). (2014). El Bosque Seco Tropical en Colombia. Bogotá, Colombia: Ediprint Ltda.

Pizano, C., González-M. R., González, M., Castro-L. F., López, R., Rodríguez, N., ... Toro, J. L. (2014). Las Plantas de los Bosques Secos de Colombia. En C. Pizano \& H. García (Eds.), El Bosque Seco Tropical en Colombia (pp. 49-93). Bogotá, Colombia: Ediprint Ltda.

Rangel-Ch., J. O., Cortés, D., \& Carvajal, J. E. (2012). Los municipios de Córdoba. En J. O. Rangel-Ch., J. Aguirre, \& C. Rodríguez (Eds.), La Biodiversidad de Municipios de la región Caribe de Colombia (pp. 409-694). Bogotá, Colombia: ARFO Editores e Impresiones Ltda.

Rivera, O. (2010). Flora. En J. O. Rangel-Ch. (Ed.), Colombia Diversidad Biótica IX: Ciénagas de Córdoba: Biodiversidad, Ecología y manejo ambiental (pp. 121-205). Bogotá, Colombia: ARFO Editores e Impresiones Ltda.

Rivera, O., \& Rangel-CH., J. O. (2012). Diversidad de espermatofitos de la región Caribe colombiana. En J. O. Rangel-Ch. (Ed.), Colombia Diversidad Biótica XII: La región Caribe de Colombia (pp. 199-317). Bogotá, Colombia: Editorial Códice Ltda.

Rodríguez, G., Banda, K., Reyes, S., \& Estupiñan, A. C. (2012). Lista comentada de las plantas vasculares de bosques secos prioritarios para la conservación en los departamentos de Atlántico y Bolívar (Caribe colombiano). Biota Colombiana, 13(2), 7-39.

Sanmartín, D., Angarita, D., \& Mercado, J. (2016). Estructura y composición florística del bosque seco tropical de Sanguaré-Sucre (Colombia). Ciencia en Desarrollo, 7(2), 43-56.

Unión Internacional para la Conservación de la Naturaleza - UICN. (2015). Especies para restauración. Recuperado de http://www.especiesrestauracion-uicn.org/ index.php

Uribe, A., Velásquez, P., \& Montoya, M. (2001). Ecología de poblaciones de Attalea butyraceae (Arecaceae) en un área de bosque seco tropical (Las Brisas, Sucre, Colombia). Actualidades Biológicas, 23(74), 33-39. 
Uslar, Y., Mostacedo, B., \& Saldias, M. (2004). Composición, estructura y dinámica de un bosque seco semideciduo en Santa Cruz, Bolivia. Ecología en Bolivia, 39(1), 25-43

Vargas, W. (2015). Una breve descripción de la vegetación, con especial énfasis en las pioneras intermedias de los bosques secos de La Jagua, en la cuenca alta del río Magdalena en el Huila. Colombia Forestal, 18(1), 47-70.

Vargas, J., González, A., Barona, E., \& Bolívar, W. (2016). Composición y estructura vegetal de fragmentos de bosque seco tropical y de dos zonas con actividad antrópica en La Dorada y Victoria, Caldas. Revista de Ciencia, 20(2), 13-60.
Vargas, W., \& Ramírez, W.(2014). Lineamientos generales para la restauración del Bosque Seco en Colombia. En C. Pizano \& H. García (Eds.), El Bosque Seco Tropical en Colombia (pp. 253-291). Bogotá, Colombia: Ediprint Ltda.

Vásquez, F., Pezo, D., Mora, J., \& Skarpe, C. (2012). Selectividad de especies forrajeras por bovinos en pastizales seminaturales del trópico centroamericano: un estudio basado en la observación sistemática del pastoreo. Zootecnia Tropical, 30(1), 63-80.

Villareal, H., Alvarez, M., Córdoba, S., Escobar, F., Fagua, G., Gast, F., ... Umaña, A. (2004). Manual de métodos para el desarrollo de inventarios de biodiversidad. Bogotá, Colombia: Panamericana Formas e Impresos S.A. 